\title{
Structured Neural Network Modelling of Multi-valued Functions for Wind Vector Retrieval from Satellite Scatterometer Measurements
}

\author{
David J. Evans and Dan Cornford and Ian T. Nabney \\ d. j.evans@aston.ac.uk
}

\begin{abstract}
A conventional neural network approach to regression problems approximates the conditional mean of the output vector. For mappings which are multi-valued this approach breaks down, since the average of two solutions is not necessarily a valid solution. In this article mixture density networks, a principled method to model conditional probability density functions, are applied to retrieving Cartesian wind vector components from satellite scatterometer data. A hybrid mixture density network is implemented to incorporate prior knowledge of the predominantly bimodal function branches. An advantage of a fully probabilistic model is that more sophisticated and principled methods can be used to resolve ambiguities.
\end{abstract}

Keywords: Wind vector retrieval; ERS-1 satellite; probabilistic models; mixture density networks; neural networks 


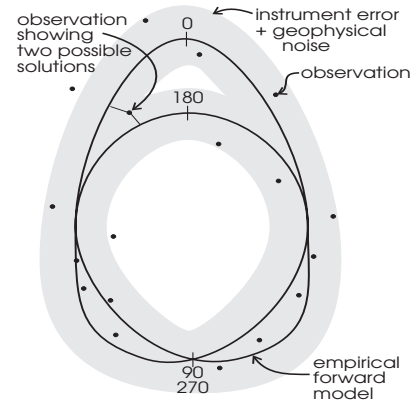

Figure 1: A two dimensional sketch of the scatterometer measurement space. The two dimensional slice is taken through the measurement manifold at constant wind speed. For a noisy observation there are at least two solutions in wind direction.

\section{Introduction}

Scatterometers carried on board satellites allow the inference of wind vectors over the ocean (Offler, 1994). There are two approaches to retrieving local wind vectors, $(u, v)$, from local scatterometer observations, $\boldsymbol{\sigma}^{o}$, using either a local empirical forward or a local empirical inverse model (Nabney et al., 1998, this issue). The forward model (Stoffelen and Anderson, 1997a) and (Ramage et $a l ., 1998$, this issue), which maps $(u, v) \rightarrow \sigma^{o}$, requires some local inversion to obtain the wind vectors. The current operational method inverts the forward model by finding an estimated $\boldsymbol{\sigma}^{\circ}$ on the forward model manifold that is closest to the observed scatterometer measurement (Stoffelen and Anderson, 1997b). The alternative approach, addressed in this paper, is to directly infer wind vectors from scatterometer data. Models of this form, mapping $\boldsymbol{\sigma}^{o} \rightarrow(u, v)$, are called inverse models. Once the local wind vectors have been inferred, either by using the forward or inverse models, a spatial prior model can be used to infer the wind field over the ocean surface (Nabney et al., 1998).

The scatterometer data is collected by the ERS-1 satellite launched in 1991 by the European Space Agency. The satellite sweeps the ocean surface in swathes approximately $500 \mathrm{~km}$ wide, sampling nineteen cells across the swathe, where the position across the swathe is given by the antenna beam incidence angle. Each cell is approximately $50 \mathrm{~km}$ by $50 \mathrm{~km}$, and so there is some overlapping between cells. The scatterometer has three antennae, in the same plane, pointing in different directions with respect to satellite propagation. The antennae sample each cell building up a measurement triplet, $\boldsymbol{\sigma}^{o}$. It is possible to determine the local wind vector for each cell using the scatterometer observations and the incidence angles of the antennae beams (Offler, 1994).

Previous work (Long and Mendel, 1991) has shown that there is a unique set of wind vectors called the noisy ambiguity set which is identifiable from a single scatterometer measurement, that is, the inverse mapping exists and is multi-valued. The multi-valued solution of the inverse mapping arises largely from noise on the observations. This is illustrated in Fig. 1, a sketch of a two dimensional slice through the three dimensional measurement space. The position of the observation on the model manifold is a function of wind speed and direction (Stoffelen and Anderson, 1997a). A noisy observation is unlikely to lie on the model manifold making it uncertain from which of the two model branches the observation originates. Thus there are at least two solutions for wind direction from a single scatterometer observation. These two solutions are roughly $180^{\circ}$ apart in wind direction, and are generally referred to as the ambiguous solutions (Stoffelen and Anderson, 1997b). 


\section{$1.1 \quad$ Background}

Novel neural network approaches have been applied to wind retrieval from scatterometer observations. In (Thiria et al., 1993) neural networks were used to infer wind direction and speed directly from simulated scatterometer data. For each incidence angle, the model consisted of two feed forward neural networks. One network uniquely modelled wind speed, the other, modelled wind direction by classifying the wind direction into thirty six bins representing ten degree intervals. The inputs to the neural network took neighbourhood information from the surrounding cells giving spatial information. In addition to the scatterometer data the wind direction network also took wind speed as an input. Simulated data was used because ERS-1 was not operational. The results showed neural networks to be a promising avenue of investigation for a solution to this inverse problem. In (Richaume et al., 1998) the models of (Thiria et al., 1993) are trained using data collected from ERS-1. Performance of the models in (Richaume et al., 1998) is shown to improve upon results obtained by the operational wind retrieval system at the European Space Agency.

In (Cornford et al., 1997) wind speed was modelled using a multi-layer perceptron while the wind direction was modelled by a mixture density network with circular normal kernel densities (Bishop and Nabney, 1996) to model the full conditional probability density of the wind direction given the scatterometer measurements. In addition to the scatterometer measurements, the incidence angle of the mid beam antenna was included as an input to the networks. The wind speed model performed within the designed specification of the instrument of $2 \mathrm{~ms}^{-1}$. For wind direction, the models learned the inherent ambiguity in the problem, but did not perform as well as the models of (Richaume et al., 1998).

In (Stoffelen, 1998) it is shown that it is preferable to analyse wind vector components in Cartesian co-ordinates rather than wind speed and direction (polar co-ordinates), as the noise distribution on the the predicted wind vector components is shown to be spherically Gaussian. In this paper we use this information and directly model the Cartesian wind vector components from scatterometer observations for the first time.

\section{Modelling multi-valued functions}

\subsection{Theory of mixture density networks}

Mixture Density Networks (MDNs) provide a framework for modelling conditional probability density functions, denoted $P(\mathbf{t} \mid \mathbf{x})$ (McLachlan and Bashford, 1988; Bishop, 1995). The distribution of the $c$-dimensional outputs, $\mathbf{t} \in R^{c}$, is described by a parametric model whose parameters are determined by the output of a neural network, which takes $\mathbf{x}$ as its inputs. The general model is described by:

$$
P(\mathbf{t} \mid \mathbf{x})=\sum_{j=1}^{M} \alpha_{j}(\mathbf{x}) \phi_{j}(\mathbf{t} \mid \mathbf{x})
$$

and

$$
\sum_{j=1}^{M} \alpha_{j}(\mathbf{x})=1
$$

Where $\alpha_{j}(\mathbf{x})$ represents the mixing coefficients (which depend on $\left.\mathbf{x}\right), \phi_{j}(\mathbf{t} \mid \mathbf{x})$ are the kernel distributions of the mixture model (whose parameters also depend on $\mathbf{x}$ ), and $M$ is the number of 
kernels in the mixture model. Generally the kernels used are $c$-dimensional spherical Gaussians of the form:

$$
\phi_{j}(\mathbf{t} \mid \mathbf{x})=\frac{1}{(2 \pi)^{\frac{c}{2}} \sigma_{j}^{c}(\mathbf{x})} \exp \left(-\frac{\left\|\mathbf{t}-\boldsymbol{\mu}_{j}(\mathbf{x})\right\|^{2}}{2 \sigma_{j}^{2}(\mathbf{x})}\right)
$$

In principle a Gaussian mixture model with kernels of the type given by (3) can approximate any density function providing the parameters are chosen correctly (McLachlan and Bashford, 1988). It follows then that for any given value of $\mathbf{x}$, the mixture model (1) can model the conditional density function $P(\mathbf{t} \mid \mathbf{x})$. To achieve this the parameters of the mixture model ${ }^{1}$ are taken to be general continuous functions of $\mathbf{x}$. These functions are modelled as the outputs of a conventional neural network that takes $\mathbf{x}$ as its input. It is this combination of a Gaussian mixture model, whose parameters are dependent on the output of feed forward neural that takes $\mathbf{x}$ as its inputs, that is referred to as a Mixture Density Network and is represented schematically in Fig. 2.1.

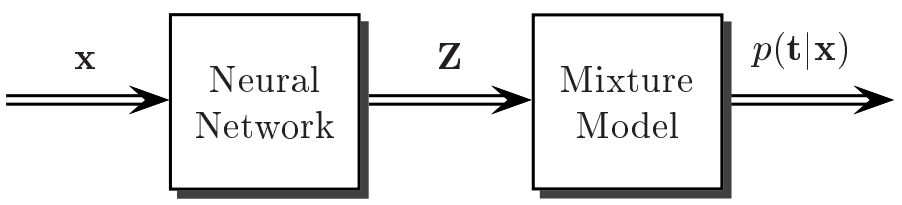

Figure 2: The structure of a Mixture Density Network. The inputs $\mathbf{x}$ are feed through a neural network. The outputs of the neural network, $\mathbf{Z}$, define the parameters of the Gaussian mixture model

By choosing sufficient kernels in the mixture model and a neural network with sufficient hidden units the MDN can approximate as closely as desired any conditional density, $P(\mathbf{t} \mid \mathbf{x})$ (Bishop, 1995). The neural network element of the MDN is implemented with a standard Multi-Layer Perceptron (MLP) with single hidden layer of tanh units and an output layer of linear units.

\subsection{Modelling the geophysical problem}

In the context of this application each input pattern for the MDN, $\mathbf{x}$, is the observed scatterometer data, $\boldsymbol{\sigma}^{o}$ and the cosine of the incidence angle, $\theta$. Modelling the wind vector components directly implies that the targets of the MDN, $\mathbf{t}$, are the wind vector components $(u, v)$. The general description of the MDN, (1), is then re-expressed using geophysical parameters as:

$$
P\left(u, v \mid \boldsymbol{\sigma}^{o}, \theta\right)=\sum_{j=1}^{M} \alpha_{j}\left(\boldsymbol{\sigma}^{o}, \theta\right) \phi_{j}\left(u, v \mid \boldsymbol{\sigma}^{o}, \theta\right)
$$

\subsection{Modelling the inherent geophysical knowledge}

The MDN architecture is modified to to model the known geophysical knowledge of the problem, the $180^{\circ}$ ambiguity in wind direction. The hybrid MDN has two kernels. One kernel is free to

\footnotetext{
${ }^{1}$ Choosing a spherical Gaussian kernel determines the parameters to be the mixing coefficients and the variances and centres (or means) of the kernel functions.
} 


\begin{tabular}{|c|c|c|c|}
\hline \multicolumn{2}{|c|}{ MDN architecture } & \multirow{2}{*}{$\begin{array}{l}\text { Vector RMS } \\
\text { errors }\end{array}$} & \multirow{2}{*}{$\begin{array}{l}\text { Percentage } \\
\text { within } 20^{\circ}\end{array}$} \\
\hline Kernels & Hidden Units & & \\
\hline 2 (Hybrid) & 35 & 4.33 & 73.38 \\
\hline 2 (Hybrid) & 50 & 4.18 & 70.32 \\
\hline 2 & 35 & 4.02 & 72.76 \\
\hline 2 & 50 & 4.03 & 74.10 \\
\hline 4 & 20 & 3.82 & 76.76 \\
\hline 4 & 25 & 3.69 & 76.82 \\
\hline 4 & 30 & 3.90 & 76.64 \\
\hline 4 & 35 & 3.89 & 76.94 \\
\hline 4 & 50 & 3.73 & 77.12 \\
\hline 4 & 90 & 4.29 & 76.64 \\
\hline 12 & 35 & 4.58 & 76.74 \\
\hline 12 & 50 & 4.24 & 77.16 \\
\hline
\end{tabular}

Table 1: Results of the fourteen MDN configurations. These results are generated from a test data set of 5000 examples.

move, the other is positioned diametrically opposite the first in $(u, v)$ space, by taking the negative mean of the free moving kernel. The simplified model becomes:

$$
P(\mathbf{t} \mid \mathbf{x})=\alpha(\mathbf{x}) \phi(\mathbf{t} \mid \mathbf{x})+(1-\alpha(\mathbf{x})) \psi(\mathbf{t} \mid \mathbf{x})
$$

where the kernels are defined by diametrically opposed spherical Gaussians with common variances:

$$
\begin{aligned}
\phi(\mathbf{t} \mid \mathbf{x}) & =\frac{1}{2 \pi \sigma^{2}(\mathbf{x})} \exp \left(-\frac{\|\mathbf{t}-\boldsymbol{\mu}(\mathbf{x})\|^{2}}{2 \sigma^{2}(\mathbf{x})}\right) \\
\psi(\mathbf{t} \mid \mathbf{x}) & =\frac{1}{2 \pi \sigma^{2}(\mathbf{x})} \exp \left(-\frac{\|\mathbf{t}+\boldsymbol{\mu}(\mathbf{x})\|^{2}}{2 \sigma^{2}(\mathbf{x})}\right)
\end{aligned}
$$

\section{Results}

In total twelve networks were trained ${ }^{2}$ taking inputs $\left(\boldsymbol{\sigma}^{o}, \theta\right)$. The performance of the networks is evaluated using the vector Root Mean Square (RMS) error between the predicted and target values on a test data set and the percentage of predicted directions from the two most probable modes that fall within twenty degrees of the target wind direction. The results are summarised in Table 1.

The results suggest that model performance is more sensitive to the number of kernels in the MDN configuration than the number of hidden units in the MLP.

\subsection{Discussion}

The complexity of the mapping, $\left(\boldsymbol{\sigma}^{o}, \theta\right) \rightarrow(u, v)$, is modelled by the MLP part of the MDN. The focus of the investigation is on MDNs with four kernels. Here the difference in the performance of percentage within $20^{\circ}$ between the best and worst model is less than $0.5 \%$, and for vector RMS error is $0.6 \mathrm{~ms}^{-1}$. It seems possible these differences are due to different initial positions on the error surface. The model with ninety hidden units does not perform as well for vector RMS error,

\footnotetext{
${ }^{2}$ When training the MDNs, the inputs are assumed to be noiseless in comparison to the noise on the targets.
} 


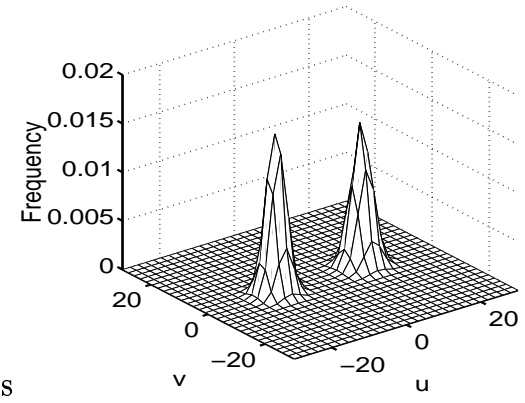

(a) Strongly bimodal

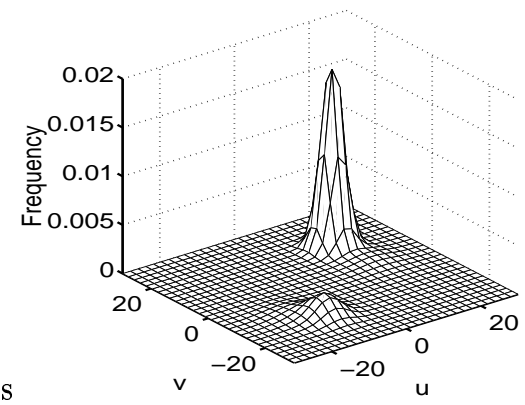

(b) Bimodal, but one mode dominating.

Figure 3: The conditional probability distribution of the wind vectors $(u, v)$ given the scatterometer data for a MDN with 4 kernels, 20 hidden units

and it is suggested that this is due to the model over-fitting. The model with twenty hidden units gives a good indication of the complexity of the mapping, $\left(\boldsymbol{\sigma}^{o}, \theta\right) \rightarrow(u, v)$.

Comparing the hybrid MDNs with the MDNs with two kernels it is interesting to note that the directional performance is similar, and the vector RMS differs by less than $0.3 \mathrm{~ms}^{-1}$ between best and worst case. This gives strong evidence to suggest the solution is dominantly bimodal (see Fig. 3 ) with these models being approximately $180^{\circ}$ apart in direction. However the models with four kernels out-perform those models with two. The complexity of the density model in the MDN is related to the number of kernels in the Gaussian mixture model. The improved performance of the MDNs with four kernels is attributed to two factors. Firstly, although the results suggest that the modes of the conditional distribution are dominantly bimodal, they are not always Gaussian or spherically symmetric, suggesting that the noise on the targets is heavier tailed than originally assumed. Four kernels in the MDN are able to model the non-Gaussian, non-spherical modes in the conditional probability distribution more efficiently than those with two kernels. Secondly, the increased flexibility of four kernels permits the MDN to place kernels into four quadrants of $(u, v)$ when appropriate, flexibility which is not available to the models with two kernels. Two further experiments obtained results for MDNs with twelve kernels. The results show that there is an increase in the vector RMS error. This is due to the model over-fitting, since it has sufficient flexibility to model both the underlying data generator and the noise on the training data set, and hence yields poor results for the test set.

Bench marking against previous work is difficult because of the different data sets used when training and testing the models. However, bearing this in mind, it is useful to compare these results with other work. Previous work by (Cornford et al., 1997), which model each cell independently, achieved a correct solution within $20^{\circ}$ roughly $73 \%$ of the time when considering the two most probable solutions. The results reported in (Richaume et al., 1998), achieve a correct solution more than $85 \%$ of the time in wind direction when considering the two most probable solutions. However in (Richaume et al., 1998) it must be noted that spatial information is also provided at the inputs to the networks, which is believed to provide additional disambiguation skill (Cornford et al., 1997).

The results of this study improved on the results of the local models in (Cornford et al., 1997). When the local models trained in this study are applied using the methods proposed in (Nabney et al., 1998, this issue) it is hoped that we can further improve performance. Models of the form (Richaume et al., 1998) which use a spatial context at their inputs cannot be used with prior wind-field models (Nabney et al., 1998) 


\section{Conclusions}

In this paper a novel method for modelling the Cartesian wind vector components, $(u, v)$, directly from scatterometer data has been introduced. By using the MDN framework, a fully probabilistic model, $P\left(u, v \mid \boldsymbol{\sigma}^{o}, \theta\right)$, has been developed which describes the joint probability distribution of the wind vectors given the scatterometer observations. The hybrid MDN has shown that the solution is dominantly bimodal, agreeing with earlier work (Stoffelen and Anderson, 1997b). Training MDNs with several different architectures suggests that these are the best results achievable, given the data, by local modelling of the inverse mapping $\left(\boldsymbol{\sigma}^{o}, \theta\right) \rightarrow(u, v)$.

Further work using improved data selection techniques for generating training data sets together with the use of committees of networks (Bishop, 1995, p 364) is expected to improve the model performance.

\section{Acknowledgements}

This work is supported by the European Union funded NEUROSAT programme (grant number ENV4 CT96-0314).

\section{References}

Bishop, C. M. 1995. Neural Networks and Pattern Recognition. Oxford University Press.

Bishop, C. M. and I. T. Nabney 1996. Modelling Conditional Probability Distributions for Periodic Variables. Neural Computation 8, 1123 - 1133.

Cornford, D., I. T. Nabney, and C. M. Bishop 1997. Neural Network based Wind Vector Retrieval from Satellite Scatterometer Data. Neural Computing and Applications. submitted.

Long, D. and J. M. Mendel 1991. Identifiability in Wind Estimation from Scatterometer Measurements. IEEE Transactions on Geoscience and Remote Sensing 29, 268 - 276.

McLachlan, G. J. and K. E. Bashford 1988. Mixture Models: Inference and Applications to Clustering. New York: Marcel Dekker.

Nabney, I. T., D. Cornford, and C. K. I. Williams 1998. Bayesian Inference for Wind Field Retrieval. Neurocomputing Letters. submitted.

Offler, D. 1994. The Calibration of ERS-1 Satellite Scatterometer Winds. Journal of Atmospheric and Oceanic Technology 11, $1002-1017$.

Ramage, G., D. Cornford, and I. T. Nabney 1998. A Neural Network Sensor Model with Input Noise. Neurocomputing Letters. submitted.

Richaume, P., F. Badran, M. Crepon, C. Mejia, H. Roquet, and S. Thiria 1998. Neural Network Wind Retrieval from ERS-1 Scatterometer Data. Journal of Geophysical Research.

Stoffelen, A. 1998. Toward the True Near Surface Wind Speed: Error Modeling and Calibration Using Triple Location. Journal of Geophysical Research 103, 7755 - 7766.

Stoffelen, A. and D. Anderson 1997a. Scatterometer Data Interpretation: Estimation and Validation of The Transfer Function CMOD4. Journal of Geophysical Research 102, 5767 5780 .

Stoffelen, A. and D. Anderson 1997b. Scatterometer Data Interpretation: Measurement Space and Inversion. Journal of Atmospheric and Oceanic Technology 14, 1298 - 1313. 
Thiria, S., C. Mejia, and F. Badran 1993. A Neural Network Approach for Modelling Nonlinear Transfer Functions: Application for Wind Retrieval from Spaceborne Scatterometer Data. Journal of Geophysical Research 98, 22827 - 22841. 